\title{
Narration und Materialität im Bilderbuch
}

Narration et matérialité dans les livres d'images

Narration and Materiality in Picturebooks

\section{Rita FINKBEINER}

\section{OpenEdition}

\section{Journals}

Édition électronique

URL : http://journals.openedition.org/ceg/4046

DOI : $10.4000 /$ ceg.4046

ISSN : 2605-8359

\section{Éditeur}

Presses Universitaires de Provence

Édition imprimée

Date de publication : 15 novembre 2018

Pagination : 201-214

ISBN : 979-10-320-0183-7

ISSN : 0751-4239

\section{Référence électronique}

Rita FINKBEINER, «Narration und Materialität im Bilderbuch », Cahiers d'Études Germaniques [Online], 75 | 2018, Online erschienen am: 25 April 2020, abgerufen am 25 November 2020. URL : http:// journals.openedition.org/ceg/4046 ; DOI : https://doi.org/10.4000/ceg.4046 


\title{
Narration und Materialität im Bilderbuch
}

\author{
Rita FINKBEINER \\ Johannes Gutenberg-Universität Mainz
}

\section{Einleitung}

Bilderbücher sind seit etwa drei Jahrzehnten ein etablierter Gegenstand akademischer Forschung. ${ }^{1}$ Sie werden nicht nur von der wachsenden LiteracyForschung untersucht, die sich mit dem Erwerb von Lese- und Schreibfähigkeiten und, etwas weiter gefasst, mit dem Literatur-, Bild- und Medienerwerb beschäftigt. ${ }^{2}$ Bilderbücher sind auch Gegenstand literaturwissenschaftlicher, semiotischer und kunsthistorischer Ansätze. ${ }^{3}$ Diese rücken Fragen nach der spezifischen Ästhetik von Bilderbüchern in den Vordergrund. In interaktionslinguistischen und entwicklungspsychologischen Studien wird die Bedeutung des gemeinsamen Betrachtens von Bilderbüchern z.B. für den Worterwerb ${ }^{4}$ oder den Erwerb von interaktionalen Fähigkeiten ${ }^{5}$ herausgestellt.

Einen Großteil seiner Faszination verdankt das Bilderbuch seinem multimodalen Charakter. Während Bildern in Kinder- und Jugendbüchern traditionell eher eine illustrierende Funktion und damit eine dem Text untergeordnete Rolle zugeschrieben wurde, herrscht in der modernen Bilderbuchforschung Einigkeit darüber, dass die Charakteristik des Bilderbuchs gerade in der intensiven Wechselbeziehung von Text und Bild besteht. ${ }^{6}$ Im

1. Einen entscheidenden Impuls für die Bilderbuchforschung gab die einflussreiche Arbeit von Nodelman, vgl. Perry Nodelman, Words about Pictures: The Narrative Art of Children's Picture Books, Athens, GA, University of Georgia Press, 1988.

2. Bettina Kümmerling-Meibauer, Jörg Meibauer, Kerstin Nachtigäller et al. (Hrsg.), Learning from picturebooks. Perspectives from child development and literacy studies, London, Routledge, 2015; Eva Gressnich, Claudia Müller, Linda Stark (Hrsg.), Lernen durch Vorlesen. Sprach- und Literaturerwerb in Familie, Kindergarten und Grundschule, Tübingen, Narr, 2016.

3. Maria Nikolajeva, Carole Scott, How Picturebooks Work, New York/ London, Routledge, 2001; Sophie Van der Linden, Lire l'album, Le Puy-en-Velay, L'Atelier du poisson soluble, 2007; Evelyn Arizpe, Maureen Farrell, Julie McAdams (Hrsg.), Picturebooks: Beyond the Borders of Art, Narrative, and Culture, New York, Routledge, 2013.

4. z.B. Jessica Horst, „Word learning via shared storybook reading“, in Kümmerling-Meibauer et al. (Hrsg.), Learning from picturebooks, S. 181-193.

5. z.B. Katharina Rohlfing, Angela Grimminger, Kerstin Nachtigäller, „Gesturing in joint book reading“, in Kümmerling-Meibauer et al. (Hrsg.), Learning from picturebooks, S. 99-116.

6. Nikolajeva, Scott, How Picturebooks Work, S. 1-28. 
Mittelpunkt der literaturwissenschaftlichen Bilderbuchforschung steht daher heute die Frage, wie sich im Zusammenspiel von bildlichen und verbalen Codes Bedeutung konstituiert.

Neben das Interesse an der Bild-Text-Beziehung im Bilderbuch ist in den letzten Jahren, im Zuge des "multimodalen Turns“7, auch ein wachsendes Interesse an physisch-materiellen Aspekten des Bilderbuchs getreten. Dies kann zum Teil auf Tendenzen des Bilderbuchmarktes seit Anfang des 21. Jahrhunderts zurückgeführt werden, verstärkt Bilderbücher zu produzieren, die unterschiedliche Materialien und Techniken nutzen, wie etwa Bilderbücher aus Stoff, wasserfeste Bilderbücher aus Plastik, Bilderbücher mit integrierten Geräuscheffekten und „Spielbilderbücher" unterschiedlicher Art, z.B. Zieh- und Klapp-Bilderbücher. ${ }^{8}$

Materialität im Bilderbuch wird, auch in Reaktion auf diesen Trend, in literaturdidaktischen Arbeiten v.a. unter dem Aspekt des Spielens bzw. spielenden Lernens untersucht. ${ }^{9}$ Aus Sicht der Textlinguistik kommt Materialität im Bilderbuch dagegen insbesondere in Bezug auf Fragen der Textsortenbestimmung und des Textsortenwandels in den Blick. ${ }^{10}$ Für die Narratologie schließlich spielt die Frage eine wichtige Rolle, wie Materialität auf die erzählte Geschichte bezogen ist. ${ }^{11}$ Oftmals wird hierbei davon ausgegangen, dass metafiktionale Mittel, die mit Materialität spielen, eher in Bilderbüchern für ältere Kinder eingesetzt werden. ${ }^{12}$

Im vorliegenden Beitrag gehe ich anhand von ausgewählten Fallbeispielen der Frage nach, wie Materialität zur Sinn-Genese der im Bilderbuch erzählten Geschichte beiträgt. Dabei wende ich mich gerade solchen Bilderbüchern zu, bei denen nicht ein Spielaspekt, eine besondere Papiermechanik oder eine außergewöhnliche Materialwahl im Vordergrund steht, sondern das einfache Pappbuch als materielles Objekt das Kind zu einer interaktiven Mitgestaltung der erzählten Geschichte anregt. Dabei wird sich zeigen, dass schon Bilderbücher für Kinder ab zwei Jahren die Materialität des Buches zu narrativen Zwecken nutzen, indem sie sie gezielt als metafiktionales Mittel einsetzen. Eine sich hier anschließende Frage ist, welche Implikationen die Einbindung der Materialität

7. Hans-Jürgen Bucher, „Multimodalität - ein universelles Merkmal der Medienkommunikation: Zum Verhältnis von Medienangebot und Medienrezeption“, in ders., Peter Schumacher (Hrsg.), Interaktionale Rezeptionsforschung. Theorie und Methode der Blickaufzeichnung in der Medienforschung, Wiesbaden, Springer VS, 2012, S. 51-82.

8. Bettina Kümmerling-Meibauer, „From babybooks to picturebooks for adults. European picturebooks in the new millennium“, Word \& Image 31:3, 2015, S. 249-264.

9. Tamara Al Chammas, Das Spielbilderbuch. Ästhetische Formen und Chancen frühkindlicher Förderung, Dissertation, Universität Oldenburg, 2012, [http://oops.uni-oldenburg.de/1373/1/ alcspi12.pdf], Stand: 30. August 2017.

10. Hiloko Kato, „A new generation of pop-up books“ - Alice for the iPad und die Bedeutung von Materialität und medialer Technik für den Textsortenwandel“, in Stefan Hauser, Ulla Kleinberger, Kersten Sven Roth (Hrsg.), Musterwandel - Sortenwandel. Aktuelle Tendenzen der diachronen Text(sorten)linguistik, Frankfurt a.M., Lang, 2014, S. 183-206.

11. Evelyn Arizpe, „All this book is about books: Picturebooks, Culture, and Metaliterary Awareness“, in Teresa Colomer, Bettina Kümmerling-Meibauer, Cecília Siva-Díaz (Hrsg.), New Directions in Picturebook Research, New York, Routledge, 2010, S. 69-82.

12. Kümmerling-Meibauer, „From babybooks to picturebooks for adults“, S. 253. 
in die Narration für den kindlichen Sprach- und Literaturerwerb hat. Einige vorläufige Überlegungen dazu stelle ich am Ende des Beitrags an.

\section{Erzählen im Bilderbuch}

Viele, wenn auch nicht alle Bilderbücher erzählen Geschichten. ${ }^{13}$ Erzählen lässt sich aus textlinguistischer Sicht als ein bestimmtes Vertextungsmuster beschreiben, das vielfältige sprachliche, interaktionale, situative und mediale Ausprägungen erhalten kann. ${ }^{14}$ In der Tradition der story grammar ${ }^{15}$ wie auch in Ansätzen zum Erzählerwerb ${ }^{16}$ steht meist der Erzähltext - die Geschichte selbst - im Fokus. Unter einem Erzähltext lässt sich ein kohäsiver und kohärenter Text verstehen, in dem eine Sequenz von Ereignissen dargestellt wird, die auf einen Höhepunkt bzw. eine Komplikation zusteuern, wobei eine Absicht zu erkennen ist, den Leser/ Hörer emotional zu involvieren. ${ }^{17}$

Der (geschriebene oder gesprochene) Text ist im Bilderbuch als multimodaler Kommunikationsform jedoch nur ,eine“ Dimension, in der sich die erzählte Geschichte konstituiert. Staiger ${ }^{18}$ unterscheidet insgesamt fünf Dimensionen oder Gestaltungsebenen des Bilderbuchs: (i) die narrative Dimension mit den Kategorien der histoire (Was wird erzählt?) und des discours (Wie wird erzählt?), die sowohl mit Hilfe von bildlichen als auch verbalen Codes dargestellt sein können; (ii) die bildliche Dimension, zu der alle Aspekte der visuellen Gestaltung und des Designs einschließlich der Typographie gehören; (iii) die verbale Dimension mit Parametern der sprachlichen Gestaltung; (iv) die intermodale Dimension, d.h. die Bild-Schrifttext-Interaktion; und (v) die paratextuelle und materielle Dimension, d.h. ,alles, was den eigentlichen Text umschließt“. ${ }^{19}$

Aus einer handlungsorientierten Perspektive sollte dabei mitbedacht werden - und diesen Aspekt blendet Staiger aus -, dass Bilderbücher für ganz bestimmte interaktionale Settings gemacht sind. Bilderbücher werden typischerweise in einer Situation des shared book reading rezipiert, für die das gemeinsame Betrachten, das Zeigen und das Umblättern, das Zuhören und Fragen stellen

13. Auch wenn manchmal der Eindruck erweckt wird, wie etwa bei Staiger, Bilderbücher seien (reine) Erzählmedien, so ist klar, dass es neben erzählenden Bilderbüchern eine Vielfalt weiterer Typen gibt, z.B. Konzeptbilderbücher, Sachbilderbücher und auch hybride Formen. Vgl. Michael Staiger, „Erzählen mit Bild-Schrifttext-Kombinationen“, in Julia Knopf, Ulf Abraham (Hrsg.), BilderBücher, Bd. 1: Theorie, Baltmannsweiler, Schneider-Verlag Hohengehren, 2014, S. 13.

14. Elisabeth Gülich, Heiko Hausendorf, „Vertextungsmuster Narration“, in Klaus Brinker et al. (Hrsg.), Text- und Gesprächslinguistik. Berlin, de Gruyter (HSK 16:1), 2000, S. 369-385.

15. David E. Rumelhart, „Notes on a Schema for Stories“, in Daniel G. Bobrow, Allan Collins (Hrsg.), Representation and Understanding, New York, Academic Press, 1975, S. 211-236.

16. Dietrich Boueke et al., Wie Kinder erzählen. Untersuchungen zur Erzähltheorie und zur Entwicklung narrativer Fähigkeiten, München, Fink, 1995.

17. Bettina Kümmerling-Meibauer, Jörg Meibauer, „Picturebooks and early literacy. How do picturebooks support early conceptual and narrative development?“, in Kümmerling-Meibauer et al. (Hrsg.), Learning from picturebooks, S. 18.

18. Staiger, „Erzählen mit Bild-Schrifttext-Kombinationen“, S. 12-23.

19. Ibid., S. 13. 
zentral sind. Man kann davon ausgehen, dass Bilderbücher gezielt Anregungen für die Gestaltung dieser Rezeptionssituation geben. ${ }^{20}$ Es scheint mir daher wichtig, über die Unterscheidung der verschiedenen ästhetisch-semiotischen Dimensionen hinaus auch immer die "Handlungspotentiale' mit in den Blick zu nehmen, die die einzelnen Gestaltungsebenen eröffnen. Wir werden sehen, dass dieser Aspekt gerade in Bezug auf die Materialität des Bilderbuchs eine wichtige Rolle spielt.

Was ist nun genau mit Materialität gemeint, und wie lässt sich Materialität in Bezug auf das (Erzählen im) Bilderbuch genauer fassen? In Anlehnung an den textlinguistischen Begriff bei Ulla Fix ${ }^{21}$ gehören zur Materialität alle Aspekte, die mit der Gestaltung des Textträgers zusammenhängen. In der gesprochenen Sprache betrifft dies nach Fix die Performanz im Sinne der Rhetorik, in der geschriebenen Sprache vor allem Typographie und Lesefläche. So ist die Typographie zwar einerseits Voraussetzung eines schriftlich verfassten Textes, sie ist aber andererseits selbst auch ein Zeichensystem, hat also ein Bedeutungspotential. ${ }^{22}$ Das zeigt sich z.B. beim Kochrezept, wo bereits die ganzheitliche typographische Konstellation aus Überschrift, Zutatenliste und Anweisungstext die Textsorte indiziert. Dieser Materialitätsbegriff deckt sich in etwa mit dem, was Staiger für das Bilderbuch als „bildliche Dimension“ beschreibt. Mit materiellen Aspekten im Bilderbuch können damit einerseits die visuell-bildlichen Aspekte und ihre Interaktion mit dem Schrifttext gemeint sein. Der Begriff der Materialität lässt sich aber andererseits auch im Sinn des physisch-materiellen Ortes begreifen, an dem der Text angebracht ist. Fix spricht hier von „Lokalität“ als einem weiteren Textualitätskriterium. Neben die ,Gestaltung“ des Textträgers - die „Sehfläche“23 - tritt also die ,Art' des Textträgers, z.B. Pappe oder Stein. Dabei ist nach Fix wesentlich, dass nicht das Material an sich, also nicht „Papier, Stein, Textilien schlechthin“ den Ort ausmachen, „sondern es [...] sich um geformtes, kulturell verfestigtes, ,institutionalisiertes` Material [handelt]“" ${ }^{24}$, also z.B. Pappe als Buch, Stein als Gedenkstein. Lokalität im Sinne von Fix deckt sich somit in etwa mit Staigers „paratextuelle[r] und materielle[r] Dimension“, zu der er Aspekte wie Buchformat und -größe, Seitengröße und -form oder Papiersorte zählt. ${ }^{25}$ Unter dieser Perspektive treten in Bezug auf das Bilderbuch dessen Eigenschaften als materieller Träger des multimodalen Textes in den Vordergrund, z.B. als ge-

20. z.B. Kümmerling-Meibauer et al. (Hrsg.), Learning from picturebooks; Gressnich et al. (Hrsg.), Lernen durch Vorlesen.

21. Ulla Fix, „Nichtsprachliches als Textfaktor: Medialität, Materialität, Lokalität“, Zeitschrift für Germanistische Linguistik 36:3, 2008, S. 347.

22. Hartmut Stöckl, „Typographie: Gewand und Körper des Textes - Linguistische Überlegungen zu typographischer Gestaltung“, Zeitschrift für angewandte Linguistik 41, 2004, S. 5-48; vgl. auch Jörg Hagemann, „Typographie und Textualität“, Zeitschrift für Germanistische Linguistik 41:1, 2013, S. 40-64.

23. Ulrich Schmitz, „Sehflächenforschung. Eine Einführung“, in Hajo Diekmannshenke, Michael Klemm, Hartmut Stöckl (Hrsg.), Bildlinguistik. Theorien - Methoden - Fallbeispiele, Berlin, Erich Schmidt, 2011, S. 23-42.

24. Fix, „Nichtsprachliches als Textfaktor“, S. 349.

25. Staiger, „Erzählen mit Bild-Schrifttext-Kombinationen“, S. 15. 
drucktes Buch mit festem Kartoneinband und Seiten aus fester Pappe. ${ }^{26}$ Orte im Sinne von Fix können konstitutiv für eine Textsorte sein. Das wird z.B. bei Textsorten wie Graffito oder Klappentext augenfällig. Zwar sollte man Bilderbücher sicherlich nicht als eigene Textsorte auffassen, vermitteln sie doch Texte unterschiedlichster Textsorten, z.B. fantastische Geschichten, Sprachspiele oder Sachtexte. Ich werde im Folgenden aber zeigen, dass der Ort - die Lokalität konstitutiv für die in einem Bilderbuch erzählte ,Geschichte' sein kann, dass also die Geschichte gewissermaßen nur entsteht, wenn ihre Lokalität/ Materialität bewusst in die Rezeption einbezogen wird. Aus Sicht der Literaturwissenschaft lässt sich die Einbeziehung materieller Aspekte in die narrative Sinn-Genese als metafiktionales Verfahren beschreiben. Metafiktionale Verfahren lenken die Aufmerksamkeit des Lesers auf den Text selbst als Repräsentation oder Konstrukt. Im postmodernen Bilderbuch seit den 1990er Jahren finden sich häufıg metafiktionale Verfahren, z.B. Bild-im-Bild-Motive, Stimmenvielfalt oder multiple story endings. ${ }^{27}$ Ein solches metafiktionales Verfahren ist auch die bei Genette beschriebene narrative Metalepse. ${ }^{28}$ Darunter lassen sich Erzählweisen fassen, bei denen sich verschiedene Erzähl- oder Zeichenebenen logikwidrig durchdringen, etwa im Motiv des Lesers, der in seine Lektüre ,hineinsteigt', wie in Michael Endes Die unendliche Geschichte. ${ }^{29}$ Während aber in der Unendlichen Geschichte das Buch weniger als sinnlich-greifbares Objekt, sondern eher als Repräsentationsmedium eine Rolle spielt, eröffnet das Bilderbuch gerade in seiner Materialität als festes Pappbuch vielfältige Möglichkeiten, Metalepsen umzusetzen. Im Folgenden werde ich dies an ausgewählten Bilderbüchern ${ }^{30}$ zeigen. Dabei steht die Materialität in dem zweiten hier genannten Sinn, also im Sinne von Fix Lokalität im Zentrum meiner Überlegungen.

\section{Fallstudien}

Im Folgenden arbeite ich anhand von zwei Fallstudien im Detail heraus, inwiefern durch die Materialität des Buches für die erzählte Geschichte Sinn hergestellt werden kann. Dafür ziehe ich die beiden Bücher Klopf an! von Anna-Clara

26. Daneben gibt es auch Bilderbücher aus Stoff, Holz oder Plastik sowie digitale Formen von Bilderbüchern, z.B. Bilderbuch-Apps.

27. Cherie Allan, Playing with Picturebooks. Postmodernism and the Postmodernesque. Houndmills, Palgrave Macmillan, 2012; Lawrence Sipe, Sylvia Pantaleo (Hrsg.), Postmodern Picturebooks: Play, Parody, and Self-Referentiality, New York/ London, Routledge, 2008.

28. Gérard Genette, Figures III, Paris, Seuil, 1972 (auf Deutsch in ders., Die Erzählung, München, Fink, 1988); Gérard Genette, Métalepse. De la figure à la fiction, Paris, Seuil (Poétique), 2004.

29. Sonja Klimek, „Die Metalepse in der zeitgenössischen Kinder- und Jugendliteratur. Ein paradoxes Erzählphänomen im Zeitalter der Medialisierung“, Juli. Zeitschrift für internationale Kinderund Jugendliteraturforschung 1, 2009, S. 5-22.

30. Die gewählten Bilderbücher können das hier interessierende Verfahren der Nutzung von Materialität zur Genese einer fiktionalen Geschichte gut illustrieren. Um zu beurteilen, inwiefern sie als repräsentativ für eine bestimmte Art von Bilderbuch für eine bestimmte Altersgruppe gelten können, müsste man größer angelegte Studien durchführen. Fest steht, dass solche Bücher auf dem Markt sind und vorgelesen werden. 
Tidholm und Da kommt der Wolf von Vincent Bourgeau und Cédric Ramadier heran. Beide richten sich laut Verlagsangaben an Kinder ab etwa zwei Jahren. In einem kurzen Exkurs betrachte ich abschließend ein sogenanntes mix and match book, für dessen Rezeption die materielle Gestaltung ebenfalls konstitutiv ist, das jedoch keine Geschichte erzählt.

\section{Klopf an!}

Das Buch Klopf an! von Anna-Clara Tidholm ist zuerst 1992 auf Schwedisch erschienen $^{31}$, die deutsche Ausgabe, ein Pappbuch im A5-Format, erschien 1999 im Hanser-Verlag. ${ }^{32}$ Die Geschichte, die das Buch erzählt, nimmt den kindlichen Leser mit auf eine Wanderung durch verschiedene Zimmer eines Hauses. Das vordere Buchcover zeigt ein Kind, das mit dem Rücken zum Betrachter auf einer Treppenstufe vor einer blauen Tür steht, deren Rahmen sich vor einer roten Wand abhebt. Die Treppenstufe und der zur Tür hinführende, links und rechts von Grün eingefasste Fußweg deuten darauf hin, dass es sich um die Eingangstür $\mathrm{zu}$ einem Wohnhaus handelt. Aus der Kinderperspektive, der Untersicht, ist die Klinke unerreichbar weit oben. Die erste Doppelseite zeigt auf der linken Hälfte, verkleinert, die Ganzsicht eines roten Hauses mit blauer Tür, darunter den Text: „Schau, ein Haus mit einer blauen Tür! Mal sehen, wer da wohnt. Wir klopfen einfach an. Klopf, klopf!“ Die rechte Hälfte der Doppelseite zoomt auf die blaue Tür, so dass diese die Doppelseiten-Hälfte vollständig ausfüllt. Blättert man die Seite um, erscheint auf der nächsten Doppelseite ein Kinderzimmer, perspektivisch gezeichnet mit sich nach hinten verengenden Seitenwänden. Vorne links auf dem Fußboden ein Junge, der Trommel spielt. Der Text dazu ist auf beide unteren Seitenhälften verteilt und fragt: „Ist jemand da? Der kleine Michel! Haut die Trommel und kippt um - bum!“ An der Rückwand des Zimmers ist schon die nächste, eine rote Tür zu sehen. Die darauffolgende Doppelseite enthält links den Text „Und jetzt die rote Tür. Klopf, klopf!“, die rechte Hälfte zoomt wiederum auf die rote Tür. Nach dem Umblättern erscheint nun ein neues Zimmer, im Vordergrund an einem Tisch sieben Kaninchen. Der dazugehörige Text fragt: „Ist jemand da? Sieben Kaninchen, futtern Möhren und Blattsalat". Dieses Prinzip setzt sich sowohl auf der Bild- als auch auf der Textebene fort: An der perspektivisch dargestellten Rückwand des Zimmers mit den Kaninchen sieht der Betrachter erneut eine Tür, diesmal eine grüne, die nach dem Umblättern fokussiert wird, danach ein neues Zimmer, diesmal vier wilde Affen. Im Hintergrund sieht man eine weiße Tür, auf die die nächste Seite zoomt. Schließlich noch ein letztes Zimmer, darin fünf Bären, im Hintergrund wiederum eine blaue Tür. Beim nochmaligen Umblättern ist das hintere Buchcover erreicht,

31. Anna-Clara Tidholm, Knacka på!, Stockholm, Alfabeta Bokförlag, 1992.

32. Anna-Clara Tidholm, Klopf an!, München, Carl Hanser Verlag, 1999. Ins Deutsche übersetzt von Anu Stohner. 
das einen Weg zeigt, der von unten links nach oben rechts verläuft und sich nach hinten hin verengt.

Die hier erzählte Geschichte entwickelt sich in enger Interaktion zwischen Text, Bild und Material. Dies wird zum einen in der Fokalisierung deutlich. Der Betrachter teilt zunächst die Perspektive des Kindes auf dem Buchcover: Er blickt auf das Kind, das ihm den Rücken zudreht und seinerseits auf die Türklinke blickt. Nach dem Umblättern ist das Kind nicht mehr zu sehen, der Betrachter selbst wird jetzt im „Wir“ der Erzählerstimme direkt angesprochen: „Komm, wir klopfen einfach an!“ Ab hier nimmt der Betrachter die Rolle des Kindes in der Geschichte ein. In einem stetigen Wechsel von Distanz und Nähe des Betrachters zu den verschiedenen Türen wird dann visuell das Durchwandern der verschiedenen Räume und eine Begegnung mit den unterschiedlichen Figuren evoziert, bis der Betrachter schließlich wieder aus dem Haus hinaustritt und mit seinem Blick dem Weg in eine unbekannte Ferne folgt.

Eine zentrale Rolle für die Illusion, dass der Leser selbst durch das Haus geht, spielt das Anklopfen. Zum Anklopfen fordert einerseits die Erzählerstimme auf: Beim ersten Mal noch explizit im Adhortativ („Wir klopfen einfach an. Klopf, klopf!"), die folgenden Male dann einfach durch die Wiederholung des reduplikativen Ausdrucks ,klopf, klopf!‘ Dieses ,klopf, klopf!‘ lässt sich einerseits als Imperativ der 2. Person Singular lesen, mit dem der Betrachter - das Kind - zum Anklopfen aufgefordert wird. Der Ausdruck kann aber auch als Inflektiv gelesen werden, der quasi-performativ eine akustische Begleithandlung repräsentiert - vergleichbar einem ,schluck!' oder ,schnarch!' im Comic. ${ }^{33}$ Zum Anklopfen lädt aber zugleich auch das feste Pappmaterial der Buchseite ein, auf der die betreffende Tür seitenfüllend abgebildet ist. Anklopfen kann also nicht nur das Kind der Textwelt an die Tür, sondern auch das zuhörende Kind auf die Pappseite. Die Tür der Textwelt öffnet sich, sobald das lesende Kind die Seite des materiellen Buches umklappt. Die Pappseite wird gleichsam zur Tür, das wiederholte Umblättern bahnt den Weg durch die einzelnen Räume. Für das Hineingleiten in die fiktionale Welt spielt das Anklopfen auf die Pappseite eine wichtige Rolle: Dieses lässt sich als Markierung der Passage von einer in die andere Welt deuten. ${ }^{34}$ Indem Elemente der materiell-konkreten Oberfläche des Buches sowie seiner medialen Blättertechnik ganz gezielt als Erzählelemente eingebaut werden, erweist sich die Materialität in Klopf an! als funktional für die Geschichte. Zugleich verweist die materielle Ebene direkt auf die Handlungsebene, indem sie Anreize liefert, in der Vorlesesituation bestimmte wiederkehrende Handlungen zu vollziehen, also etwa anzuklopfen, die Tür zu öffnen oder zuzuklappen.

33. Sebastian Bücking, Jennifer Rau, „German non-inflectional constructions as separate performatives“, in Daniel Gutzmann, Hans-Martin Gärtner (Hrsg.), Beyond expressives: explorations in use-conditional meaning, Leiden, Brill, 2013, S. 59-94.

34. Maria Nikolajeva, The Magic Code. The use of magical patterns in fantasy for children, Dissertation, Universität Stockholm, 1988. 


\section{Da kommt der Wolf}

Das Buch Da kommt der Wolf von Vincent Bourgeau und Cédric Ramadier ist 2013 in französischer Originalausgabe erschienen ${ }^{35}$, die deutsche Ausgabe erschien 2014 beim Moritz-Verlag als Pappbuch in A5-Format. ${ }^{36}$ Die Geschichte versetzt den Betrachter in die Rolle einer Figur, die von einem herannahenden Wolf bedroht wird. Unten auf dem Buchcover ist auf einem waldgrünen Streifen sehr klein ein Wolf zu sehen, der auf den Betrachter ausgerichtet ist. Der Buchtitel kündigt groß an: Da kommt der Wolf! Auf der ersten Doppelseite nimmt der Waldboden die ganze untere Hälfte der Doppelseite ein, der Blick des Betrachters geht weit nach hinten in die Landschaft. Links sieht man zwei kleine Tannen, daneben wieder den Wolf, der, noch weit entfernt, auf den Betrachter zukommt. Auf der rechten Hälfte der Doppelseite findet sich im Grün des Waldbodens der Text „Schnell! Blätter um, dann bist du ihn los!“ Die nächste Doppelseite zeigt den Wolf etwas größer und mittig auf der linken Hälfte der Seite, darüber den Text „Er kommt näher ...“ Unten rechts findet sich erneut die Anweisung „Schnell, blätter um!“ Auf der nächsten Seite erscheint der Wolf noch etwas größer, schon fast auf Augenhöhe mit dem Betrachter, darüber der Text „Jetzt ist er schon ganz nah!“, dazu die Aufforderung „Schnell! Kipp das Buch nach rechts und blätter um!“ Nach dem Umblättern sieht man den Waldboden schräg von links nach rechts abfallen, darauf der Wolf, wie er nach rechts rutscht, dabei immer noch den Betrachter fixierend. Der Begleittext über dem Bild sagt „Er rutscht!“, der Text auf der rechten Seitenhälfte „Yippie! Lass das Buch gekippt und blätter um!“ Die nächste Seite zeigt den Wolf noch weiter nach rechts rutschend, schon fast das Gleichgewicht verlierend, darüber den Text „Er rutscht noch mehr!“, und auf der rechten Seite die Anweisung „Halt es weiter schief (und blätter um!)“. Auf der nächsten Doppelseite ist links nur noch der schräg abfallende Waldboden zu sehen, der Wolf ist auf die rechte Seitenhälfte gerutscht und rollt dort auf den sich jetzt am Ende des Waldbodens auftuenden Abgrund zu. Auf der linken oberen Hälfte findet sich der Text „Jetzt rollt er!“, auf der rechten unteren Hälfte „Halt das Buch noch immer schief, dann stürzt er in die Tiefe! (und blätter um)“. Blättert man um, erscheint nun über die fast ganz vom Grün ausgefüllte Doppelseite die Seitenansicht des Waldbodens, an dessen rechts in die Tiefe abfallender Seite ein Ast herausragt, an dem sich der Wolf festhält. Die Erzählerstimme ruft „0 nein! Jetzt hängt er an einem Ast!“ und instruiert uns: „Blätter um und schüttel das Buch!“ Nach dem Umblättern die Enttäuschung: „So ein Teufelskerl - er lässt nicht locker!“, dazu ein mit comicartigen speed lines und versetzten Schatten versehenes Bild des wackelnden, aber immer noch sich festklammernden Wolfes. Der Betrachter wird aufgefordert: „Blätter um und dreh das Buch auf den Kopf!“ Befolgt man diese Anweisung, sieht man nun den Wolf, der sich einfach mitgedreht hat. Die Erzählerstimme kommentiert:

35. Vincent Bourgeau, Cédric Ramadier, Au secours voilà le loup, Paris, L'école des loisirs, 2013.

36. Vincent Bourgeau, Cédric Ramadier, Da kommt der Wolf, Frankfurt a.M., Moritz-Verlag, 2014. Aus dem Französischen übersetzt von Markus Weber. 
„Er hält sich wirklich ganz doll fest, dieser Wolf!“, und fordert: „Dreh das Buch wieder zurück und blätter um!“ Auf der nächsten Doppelseite sieht man den Wolf nun wieder auf dem grünen Waldboden, schon bedrohlich nah, wie er auf den Betrachter zukommt. Eine spiralartig geschwungene Linie deutet an, dass er den Ast losgelassen hat und wieder auf den Waldboden gesprungen ist. Der Begleittext sagt „O nein ... Er ist wieder oben!“ und fordert den Betrachter auf: „Schnell, blätter um!“ Es folgt die letzte Doppelseite, darauf links, nun in seitenfüllender Größe, der Wolf, der die Zähne bleckt und noch einen Schritt auf den Betrachter zu macht. „Da ist er!“, ruft die Erzählerstimme, und „Hilfe! Klapp das Buch zu!“ Das hintere Buchcover schließlich, ganz in grün, enthält nur den Text: „Puuh! Der Wolf ist weg! Fangen wir gleich nochmal von vorne an?“

Auch in diesem Buch wird der Betrachter gleichsam zu einer Figur der erzählten Geschichte. Auf der sprachlichen Ebene spielen dafür die Imperativsätze eine wichtige Rolle, durch die der Erzähler dem betrachtenden Kind direkte Anweisungen gibt. Die Bildebene zeigt einen durchgehend auf den Betrachter ausgerichteten Wolf, der von Seite zu Seite immer größer wird, wodurch der Eindruck des Näherkommens entsteht. Die Interaktion mit dem materiellen Buch ist es schließlich, durch die sich die fiktionale Geschichte entwickelt. Der Wolf wird durch verschiedene Manöver in seinem Näherkommen gestört, lässt sich aber nicht abschütteln und kann am Ende nur durch Zuklappen gestoppt werden. Auf der Ebene der Chronologie der Erzählung werden markante Punkte durch die Materialität des Buches markiert. So beginnt der Weg des Wolfes auf dem vorderen Buchcover und endet auf dem hinteren. Zuklappen beendet seine beängstigende Annäherung, Zurückdrehen des Buches auf die Vorderseite lässt den Wolf wiederkommen. Da kommt der Wolf macht so das Blättern selbst zum Thema und geht noch weiter, indem gezeigt wird, was man sonst noch so mit einem Buch machen kann: Schütteln, auf den Kopf stellen, zuklappen. Die materielle Seite des Buches wird gezielt in den Fokus gerückt, um einen Anlass zur eigenen kreativen Mitgestaltung der im Buch erzählten Geschichte zu schaffen. ${ }^{37}$ In der Zusammenschau der hier untersuchten Bücher lässt sich festhalten, dass beide ganz gezielt metafiktionale Techniken einsetzen, die zu einer Durchdringung der fiktionalen Welt mit der Welt des lesenden Kindes führen. Für diese Durchdringung der beiden Welten spielt in beiden Büchern ihre physisch-materielle Seite eine entscheidende Rolle: Der Erzähltext thematisiert das Buch als Objekt, so dass Buch-Objekt und erzählte Geschichte ineinander übergehen. Die Materialität ist dabei jedoch nicht an sich bedeutsam. Sie wird es erst dadurch, dass sie zu einer Interaktion mit dem Buch anregt, durch die sich die Geschichte entfaltet. Narration wird damit erfahrbar als Performanz, als ein partizipatorischer, soziale Beziehungen stiftender Prozess. ${ }^{38}$

37. So kann das Kind z.B. das Tempo, in dem die einzelnen Sequenzen aufeinander folgen, selbst bestimmen, indem es schneller oder langsamer umblättert, schüttelt, dreht usw.

38. Nina Tecklenburg, Performing Stories. Erzählen in Theater und Performance, Bielefeld, transcript, 2011. 


\section{Exkurs: Mix and match book}

Die Fallanalysen haben gezeigt, dass die Ebene der Materialität auf unterschiedliche Weise zur Entfaltung der im Bilderbuch erzählten Geschichte beitragen kann. Eine interessante Beobachtung ist, dass es dazu keiner besonderen Ausstattung - etwa Geräuscheffekte, integrierte „Fühl-Items“ oder Zieh-Elemente - bedarf. Vielmehr genügt die einfache Thematisierung des (Papp-)Buches als Objekt.

Ich möchte zum Abschluss kurz vergleichend auf einen Bilderbuchtyp eingehen, der sich durch eine etwas aufwendigere Materialgestaltung auszeichnet. Dieser Bilderbuchtyp ist im Englischen unter den Bezeichnungen mix and match book oder flip-flap book bekannt. Im Deutschen ist dafür die eher unspezifische und ambige Bezeichnung „Klapp(en)bilderbuch“ gängig. ${ }^{39}$ Ein Beispiel ist das Buch Krogufant von Sara Ball. ${ }^{40}$ Mix and match books erzählen keine Geschichte, sondern vermitteln Konzepte bzw. Wörter. Es sind Bücher aus fester Pappe im Hoch- oder Querformat, deren Seiten meist dreigeteilt sind. Damit ergibt sich eine Aufteilung einer Seite in drei Klappen, die unabhängig voneinander umgeblättert werden können. Die Klappen auf der einen Hälfte der Doppelseite enthalten Wörter oder Wortteile, die auf der anderen Seite Bilder bzw. Bildteile. Häufig sind, wie in Krogufant, Tiere abgebildet. Je nachdem, wie man die einzelnen Klappen zusammenstellt, entstehen neue Tiere und neue Tierbezeichnungen, z.B. eben ein „Kro-gu-fant“ mit Krokodilskopf, Jaguarbauch und Elefantenbeinen, oder ein „Wild-pan-dil“, aus „Wildente“, „Schimpanse“ und „Krokodil“. Die Möglichkeit der immer neuen Kombination operiert auf dem konstanten Schema von Dreisilbigkeit des Wortes, Dreiteilung des Tieres, Korrespondenz zwischen Silbenposition und Körperteil und Klappenmechanik. Damit besteht die Rezeptionsleistung beim mix and match book primär im kreativen Hervorbringen eines neuen Konzepts. Für diese kreative Leistung ist die Materialität des mix and match books konstitutiv: Erst indem das Buch in seiner materiellen Aufmachung Klappen anbietet, stellt es die verschiedenen Kombinationsmöglichkeiten zur Verfügung und fordert gleichsam dazu auf, sie zu nutzen. Mix and match books verkörpern in ihrer Materialität die paradigmatische Grundform, indem sie auf dem Prinzip der Wiederholung mit Ersetzung basieren. Die Vielzahl der Kombinationsmöglichkeiten verlangt wiederum ein wiederholtes Spiel mit den Klappen. Kein Kind würde lediglich eine Klappe umblättern und das Buch dann weglegen. Hierin liegt ein Verweis auf die Rezeptionssituation - diese ist angelegt als Spiel, das beliebig wiederholt werden kann (und soll).

Während Klopf an! und Da kommt der Wolf Materialität gezielt für die Entwicklung der erzählten Geschichte nutzen, nutzt Krogufant die Materialität

39. „Klapp(en)bilderbuch“ kann sich auch auf Bilderbücher mit in die Buchseiten integrierten Klappen beziehen, unter denen sich neue Bild- oder Textelemente befinden. Al Chammas spricht stattdessen von „Verwandlungsbüchern“, auch dies ein recht vager Terminus. Das zentrale Funktionsprinzip des Buchtyps ist als „mixing and matching“ m.E. am besten erfasst. Vgl. Al Chammas, Das Spielbilderbuch, S. 110.

40. Sara Ball, Krogufant, Weinheim, Beltz \& Gelberg, 2007 [Neuausgabe]. 
gezielt für die Vermittlung von Konzepten. In allen untersuchten Bilderbüchern erweist sich Materialität damit als funktional für den Inhalt bzw. die Intention des Buches. Sie unterscheiden sich darin von manchen sogenannten „Spielbüchern“, die zwar materiell aufwendig gestaltet sind, bei denen die Materialität aber nicht mit dem Inhalt des Buches korrespondiert. Ein Beispiel ist Mein erstes Kuschelbuch ${ }^{41}$ ein textloses Bilderbuch für Kinder ab drei Monaten, das als Stoffbuch gestaltet ist, und dessen dickes Stoffcover knistert, wenn man das Buch in die Hand nimmt. Dieses Knistern steht jedoch in keinem Zusammenhang zu dem, was die Cover-Abbildung zeigt, nämlich einen Bären. Der zusätzliche auditive Reiz steigert möglicherweise die Attraktivität des Buches, erscheint aber für das, was das Buch vermitteln möchte, arbiträr. ${ }^{42}$

\section{Implikationen für den Sprach- und Literaturerwerb}

Die Analysen haben gezeigt, dass Bilderbücher für Kinder ab zwei Jahren bereits gezielt metafiktionale Verfahren einsetzen, die sich die Materialität des Buches für die Entfaltung der erzählten Geschichte zunutze machen. Die in Klopf an! und Da kommt der Wolf erzählten Geschichten sind multimodale Konstrukte, die auf den Ebenen von Text, Bild und Material vielfältig strukturiert sind.

Man kann sich fragen, welche Implikationen daraus für den kindlichen Sprach- und Literaturerwerb erwachsen. Es gibt inzwischen vielfache Evidenz dafür, dass die Art der Gestaltung von Bilderbüchern Einfluss auf die Entwicklung sprachlicher und literarischer Fähigkeiten von Kindern hat. ${ }^{43}$ So ist beispielsweise bekannt, dass sogenannte Frühe-Konzepte-Bücher (early concept books), die meist textlos sind und einfache Alltagsgegenstände zeigen, Kinder in ihrem lexikalischen Erwerb unterstützen. ${ }^{44}$ Es liegt nahe, anzunehmen, dass eine besondere materielle Ausstattung dabei das Verständnis in besonderer Weise fördern kann, beispielsweise wenn durch die Integration eines weichen Fellstückchens in das Bild einer Katze eine zusätzliche haptische Erfahrung vermittelt und damit das Verstehen des Konzepts „weiches Fell“ gefördert wird. Vom gezielten Einsatz der Klappenmechanik im mix and match book sind

41. Monika Neubacher-Fesser, Mein erstes Kuschelbuch, Ravensburg, Ravensburger Verlag, 2003.

42. Pauline Liesen, „Sehen, Fühlen, Klappen. Das Spielbilderbuch für die Kleinen (0 bis 3 Jahre)“, in Bettina Kümmerling-Meibauer, Maria Linsmann (Hrsg.), Literatur im Laufstall. Bilderbücher für die ganz Kleinen, Katalog zur Ausstellung, Bilderbuchmuseum Burg Wissem Troisdorf, 2009, S. 89-98.

43. Vgl. zum Zusammenhang zwischen Spracherwerb und Kinderliteratur: Jörg Meibauer, „Spracherwerb und Kinderliteratur“, in Wolfgang Klein, Jörg Meibauer (Hrsg.), Spracherwerb und Kinderliteratur, Sonderheft der Zeitschrift für Literaturwissenschaft und Linguistik 162, 2011, S. 9-26; Rita Finkbeiner, „Phraseologieerwerb und Kinderliteratur. Verfahren der ,Verständlichmachung“ von Phraseologismen im Kinder- und Jugendbuch am Beispiel von Otfried Preußlers ,Die kleine Hexe“ und „Krabat““, Zeitschrift für Literaturwissenschaft und Linguistik 162, 2011, S. 47-73; Rita Finkbeiner, „Sprachliche Rekurrenz im kinderliterarischen Text. Eine Fallstudie zu Kirsten Boies ,Seeräubermoses““, Der Deutschunterricht 2, 2017, S. 75-81.

44. Bettina Kümmerling-Meibauer, Jörg Meibauer: „First Pictures, Early Concepts: Early Concept Books“, The Lion and the Unicorn 29, 2005, S. 324-347. 
positive Auswirkungen auf den phonologischen und morphologischen Erwerb zu erwarten. ${ }^{45}$ Hier werden Silben- und Wortstrukturen auf einzeln blätterbare Klappen abgebildet, so dass sprachliche Strukturbildung ganz konkret ,begreifbar wird. Man weiß auch, dass das gemeinsame Bilderbuchbetrachten ein routinisiertes Handlungsschema darstellt, das oft gezielt zur sprachlichen Unterweisung genutzt wird. ${ }^{46}$ Aus der Materialität des Bilderbuchs können dabei spezielle Anlässe zu einem gemeinsam gestalteten Leseerlebnis erwachsen, wenn etwa auf die Buchseite geklopft oder das Buch gedreht und geschüttelt werden soll. Aus solchen gemeinsamen Leseerlebnissen sind - neben einer unterstützenden Funktion für den Worterwerb - auch Effekte auf die literarischen Rezeptionskompetenzen von Kindern zu erwarten. ${ }^{47}$ In Bezug auf die in diesem Beitrag untersuchten Bilderbücher stellt sich vordringlich die Frage, wann und wie Kinder narrative Metalepsen verstehen lernen. Nach Klimek ${ }^{48}$ hat der Einsatz narrativer Metalepsen in der Kinder- und Jugendliteratur typischerweise nicht den Effekt, einen Illusionsbruch zu erzeugen. Metalepsen werden dort „vielmehr als wunderbare Vorgänge in Texte integriert, in denen auch sonst viel Wunderbares vorkommt, ohne dabei die Kohärenz der fiktiven Welt zu gefährden“49. Aus Sicht des Spracherwerbs stellt sich aber die Frage, wie diese Integration so einfach gelingen kann. Wie erwerben Kinder im Alter von zwei bis vier Jahren, an die sich die untersuchten Bilderbücher richten, dieses erzählerische Verfahren? Eine besondere Herausforderung liegt darin, dass für den Wort- und Konzepterwerb, der sich im Alter zwischen zwei und sechs Jahren stark entwickelt, ${ }^{50}$ die Fähigkeit ganz zentral ist, Zeichen und Referent voneinander trennen zu können. Kinder müssen also die Fähigkeit erwerben, das Bild eines Balls, bzw. den Ausdruck /ball/ vom ,realen' Ball unterscheiden zu können. In Büchern wie Klopf an! oder Da kommt der Wolf wird aber diese wichtige Trennung von Zeichen und Referent gerade aufgehoben. Die Tür der Textwelt wird zur ,realen` Tür in der Vorlesesituation, der Wolf der Textwelt wird zur ,realen' Gefahr für das zuhörende Kind. Die Aufgabe besteht also darin, eine gewisse Souveränität im Umgang mit der Zeichen/ Referent-Trennung zu entwickeln, so dass etwa für die Rezeption bestimmter Textsorten, z.B. fiktionaler Geschichten, von diesem Prinzip abgewichen werden kann. Es geht mit anderen Worten um den Erwerb von metaliterary awareness. ${ }^{51}$ Eine unterstützende Rolle kann dabei die Gestaltung der Übergänge oder Passagen spielen. So wird der Übergang von

45. Rita Finkbeiner, „Serielle Narration als Konstruktion. Studien an Bilderbüchern für Vorschulkinder", in Bettina M. Bock, Konstanze Marx, Simon Meier et al. (Hrsg.), Sprachliche Verfestigungen und sprachlich Verfestigtes, Themenheft Linguistik Online, erscheint 2018.

46. Meibauer, „Spracherwerb und Kinderliteratur“, S. 11.

47. Corinna Weis, Tanja Dinkel, „Von Pop-up-, Dreh- und Verwandlungskünsten. Faszinierende Spielbilderbücher für den Primarstufenunterricht“, in Abraham Knopf (Hrsg.), BilderBücher, S. 83-92.

48. Klimek, „Die Metalepse in der zeitgenössischen Kinder- und Jugendliteratur“, S. 7.

49. Ibid., S. 7.

50. Christina Kauschke, Kindlicher Spracherwerb im Deutschen. Verläufe, Forschungsmethoden, Erklärungsansätze, Berlin/ Boston, de Gruyter, 2012, S. 44-52.

51. Arizpe, „All this book is about books“, S. 70. 
der ,realen“ Welt in die Textwelt in Klopf an! durch das Anklopfen, d.h. durch eine taktile Erfahrung des Repräsentationsmediums markiert, in das das Kind ,eintaucht: Die genauen Effekte von Verfahren wie der narrativen Metalepse auf die sprachlichen und kognitiven Fähigkeiten von Kindern müssen aber noch viel besser empirisch erforscht werden. Dazu bedarf es einer engen Zusammenarbeit von Linguistik, Literaturwissenschaft und Kognitionsforschung.

\section{Schluss}

Die Frage, wie Narration und Materialität aufeinander bezogen sind, stellt sich für das Bilderbuch in besonderer Weise. Versteht man unter Materialität Aspekte der Gestaltung der „Sehfläche“, so rücken vor allem die vielfältigen Wechselbeziehungen zwischen Schrifttext und Bild in den Vordergrund. Hieraus ergeben sich Fragen nach dem erzähltheoretischen Status von Bildern: In welcher Weise „erzählen“ Bilder, und inwiefern ist das Erzählen mit Bildern dem Erzählen mit Sprache vergleichbar? Versteht man unter Materialität Aspekte der Art oder Beschaffenheit des Textträgers, so rückt die materielle Gestalt des Bilderbuchs als Buch, d.h. als „institutionalisiertes Material“, stärker in den Blick. Erzähltheoretisch stellt sich dann die Frage, wie die Materialität des Buches zur Sinn-Genese der Geschichte beitragen kann. Diese Frage stand im Mittelpunkt dieses Beitrags. Die Fallstudien haben exemplarisch gezeigt, wie die Materialität des Buches durch das in ihr angelegte Potential zum kreativen Handeln zur Konstitution der im Buch erzählten Geschichte beitragen kann. 
